\title{
Casimir Energy in a Conical Wedge and a Conical Cavity
}

\author{
H. Ahmedov and I. H. Duru \\ Feza Gürsey Institute, P.O. Box 6, 81220, Çengelköy, Istanbul, Turkey ${ }^{1}$.
}

\begin{abstract}
Casimir energies for a massless scalar field for a conical wedge and a conical cavity are calculated. The group generated by the images is employed in deriving the Green function as well as the wave functions and the energy spectrum.
\end{abstract}

\section{Introduction}

Image method is one of the best tools in the calculation of the Casimir energies for the polygonal regions. One can use discrete group generated by the reflections with respect to the boundaries of the polygon under consideration to obtain the Green function, wave functions and spectra of the system. The method is trivially applicable to the cavities or the given regions of space if the following conditions are fulfilled: Surfaces of the region are planar; and, they are parallel or perpendicular to each other. The discrete groups generated by reflections with respect to the planar boundaries appears to be the direct product of the translation $Z$ and the cyclic $C_{2}$ groups. The number of copies of $Z$ and $C_{2}$ depend on the dimension of the rectangle. For the parallel plate system and two dimension rectangle we have the groups $C_{2} \times Z$ and $\left(C_{2} \times Z\right)^{2}$ respectively. ( For a Casimir calculation in rectangular region we refer to the monograph [1] and references therein ).

Recently we tried to extend the image method to a set of geometries with planar boundaries without rectangular angles ${ }^{2}$. For a class of cavities with triangular crossections, making use of the tools of the group of reflections from the walls one can obtain the Green function satisfying the Dirichlet boundary conditions, then calculates the Casimir energy [3]. Point groups and crystallographic point groups play important role in constructing the Green functions and the wave functions if the region we consider is the fundamental domain of the one of those groups. In other words, if every point in the region represents different orbits of the group, the satisfaction of the required wave equation by the Green function is achieved [3], 4].

\footnotetext{
${ }^{1}$ E-mail : hagi@gursey.gov.tr and duru@gursey.gov.tr

${ }^{2}$ To our knowledge only previous example without rectangular angles was the wedge problem [2].
} 
In this work the example we present is the application of the image method to a geometry with non-planar boundary. The surface of the cone we deal with is obtained from a geometry with planar surfaces, by identifying two of these surfaces. In fact if there is an element of the point group which maps one planar boundary onto other one, we can obtain volumes with non planar boundaries. The simple example we can think of is the wedge in $R^{2}$ with the angle $\frac{2 \pi}{N}$. By identifying two lines which are the boundaries of the wedge we arrive at the conic surface with the opening angle $2 \arcsin \frac{1}{N}$. The corresponding point group is the cyclic one $C_{N}$, that is generated by the element $q$ which identifies two boundaries of the wedge. It is clear that $q^{N}=1$.

In the next section we first consider a conical wedge in $R^{3}$ with the opening angle $2 \arcsin \frac{1}{3}$. The region is the fundamental domain of the tetrahedral group which has $C_{3}$ as subgroup. We construct the Green function, then calculate the Casimir energy density for the massless scalar field.

In section III making use of the group of the previous section we construct the wave function and calculate the energy spectrum for a the conical wedge.

Section IV is devoted to the closed cone, i.e., conical cavity for which we calculate the Casimir energy which is positive.

In the last section we briefly reviewed the known Casimir energy results for 3-dimensional cavities.

The required group theoretical details are given in the appendix.

\section{Green Function and Casimir Energy in a Conical Wedge}

Consider the following three planes in the first quadrant $\left(x_{1}>0, x_{2}>\right.$ $\left.0, x_{3}>0\right)($ Figure 1$)$

$$
P_{1}: x_{1}=x_{2}, \quad P_{2}: x_{2}=0 . \quad P_{3}: x_{2}=x_{3} .
$$

We are interested in the Green function for the massless scalar field satisfying the boundary conditions

$$
\left.K(x, y)\right|_{\vec{x} \in P_{2}}=0
$$

and

$$
\left.K(x, y)\right|_{\vec{x} \in P_{1}}=\left.K(x, y)\right|_{g \vec{x} \in P_{3}}
$$

where

$$
g=\left(\begin{array}{lll}
0 & 0 & 1 \\
1 & 0 & 0 \\
0 & 1 & 0
\end{array}\right)
$$

is the rotation matrix around $\vec{n}=(1,1,1)$; i.e., the intersection of $P_{1}$ and $P_{2}$, by angle $\frac{2 \pi}{3}$. It transforms $P_{1}$ into $P_{3}$. The region under consideration

$$
S=\left\{x_{1} \geq x_{2} \geq 0, \quad x_{3} \geq x_{2} \geq 0\right\}
$$

is the fundamental domain $S=R^{3} / G$ of the group $G=T \times I$, where $T$ is the tetrahedral group and $I$ is the inversion group generated by $i=-1$ ( see 
Appendix ). Identifying planes $P_{1}$ and $P_{3}$ in the manner described in (3) we arrive at the space which is topologically equivalent to a cone with the plane $P_{2}$ being the boundary ( Figure 2 ). Bringing $\vec{x}_{1}$ onto $\vec{x}_{3}$ axis requires a rotation by the angle $\frac{2 \pi}{3}$ around $\vec{n}$. Thus the cone we obtained has an opening angle $2 \beta=2 \arcsin \frac{1}{3} \cong 39^{\circ}$. Therefore the problem in hand is equivalent to the study of the cone with Dirichlet boundary condition.

The desired Green function is of the form

$$
K(x, y)=\sum_{j=0}^{11}\left[G\left(g_{j} x, y\right)-G\left(i g_{j} x, y\right)\right]
$$

where summation runs over the 12 elements of the tetrahedral group. Here $G(x, y)$ is the free Green function for the massless scalar field in the Minkowski space ( in $\hbar=c=1$ units )

$$
G(x, y)=\frac{1}{4 \pi^{2}} \frac{1}{|x-y|^{2}} .
$$

To check the boundary conditions, we observe that for any element $g_{j} \in T$ there exist an element $g_{a} \in T$ such that $g_{a}=g_{j} g$. Therefore

$$
K(g x, y)=K(x, y)
$$

which implies that the boundary condition (13) is fulfilled. To show the boundary condition (2) we first define the reflection operators with respect to $P_{2}$

$$
q=\left(\begin{array}{ccc}
1 & 0 & 0 \\
0 & -1 & 0 \\
0 & 0 & 1
\end{array}\right)
$$

For any element $g_{j} \in T$ there exist an element $g_{b} \in T$ such that $i g_{b}=g_{j} q$. Therefore

$$
K(q x, y)=-K(x, y)
$$

which implies that the boundary condition (2) is fulfilled.

To obtain the energy momentum tensor for the conformally coupled massless scalar field we employ the well known coincidence limit formula [5]

$T_{\mu \nu}=\lim _{x \rightarrow y}\left[\frac{2}{3} \partial_{\mu}^{y} \partial_{\nu}^{x}-\frac{1}{6}\left(\partial_{\mu}^{x} \partial_{\nu}^{x}+\partial_{\mu}^{y} \partial_{\nu}^{y}\right)-\frac{\eta_{\mu \nu}}{6} \eta^{\sigma \rho} \partial_{\sigma}^{y} \partial_{\rho}^{x}+\frac{\eta_{\mu \nu}}{24} \eta^{\sigma \rho}\left(\partial_{\sigma}^{x} \partial_{\rho}^{x}+\partial_{\sigma}^{y} \partial_{\rho}^{y}\right)\right] K(x, y)$

The energy density $T(x)=T_{00}$ is given by:

$$
T(x)=\frac{1}{12 \pi^{2}} \sum_{j=1}^{11}\left[T\left(g_{j}\right)-T\left(i g_{j}\right)\right]
$$

where

$$
T(g)=\left(\frac{\operatorname{tr}(g)-1}{|\vec{\eta}|^{4}}-2 \frac{\mid\left(\left.(1+g) \vec{\eta}\right|^{2}\right.}{|\vec{\eta}|^{6}}\right)
$$

and

$$
\vec{\eta}=(1-g) \vec{x}
$$


with $g$ standing for $g_{j}$ and $i g_{j}$.

Using the invariance of $T(g)$ under $g \rightarrow g^{-1}$ we have

$$
\begin{aligned}
T\left(g_{4}\right) & =T\left(g_{5}\right) \\
T\left(g_{6}\right) & =T\left(g_{7}\right) \\
T\left(g_{8}\right) & =T\left(g_{9}\right) \\
T\left(g_{10}\right) & =T\left(g_{11}\right)
\end{aligned}
$$

The same equalities are true for elements $i g_{j}$, with $j$ running the same values as (15).

- For the rotation $g_{6}$ by angle $\frac{2 \pi}{3}$ around the line passing trough the origin and the point $(1,-1,1)$ we have

$$
\begin{gathered}
T\left(g_{6}\right)=-\frac{3}{\left(\left(x_{1}+x_{2}\right)^{2}+\left(x_{2}+x_{3}\right)^{2}+\left(x_{3}-x_{1}\right)^{2}\right)^{2}} \\
T\left(i g_{6}\right)=-\frac{6|\vec{x}|^{2}+2\left(x_{1} x_{3}-x_{1} x_{2}-x_{2} x_{3}\right.}{\left(\left(x_{1}-x_{2}\right)^{2}+\left(x_{2}-x_{3}\right)^{2}+\left(x_{3}+x_{1}\right)^{2}\right)^{3}} .
\end{gathered}
$$

Since $g_{8}$ and $g_{10}$ are rotation matrices by the same angle around the axis passing trough the origin and the points $(-1,1,1)$ and $(1,1,-1)$ we conclude that $T\left(g_{8}\right), T\left(i g_{8}\right)$ and $T\left(g_{10}\right), T\left(i g_{10}\right)$ are given by (16) and (17) with the cyclic replacements of coordinates $\left(x_{1}, x_{2}, x_{3}\right) \rightarrow\left(x_{3}, x_{1}, x_{2}\right)$ and $\left(x_{1}, x_{2}, x_{3}\right) \rightarrow\left(x_{2}, x_{3}, x_{1}\right)$ respectively.

- We also have

$$
\begin{aligned}
& T\left(g_{4}\right)=-\frac{3}{\left(\left(x_{1}-x_{2}\right)^{2}+\left(x_{2}-x_{3}\right)^{2}+\left(x_{3}-x_{1}\right)^{2}\right)^{2}} \\
& T\left(i g_{4}\right)=-\frac{6|\vec{x}|^{2}-2\left(x_{1} x_{2}+x_{1} x_{3}+x_{2} x_{3}\right)}{\left(\left(x_{1}+x_{2}\right)^{2}+\left(x_{1}+x_{3}\right)^{2}+\left(x_{2}+x_{3}\right)^{2}\right)^{3}} .
\end{aligned}
$$

- For elements satisfying the condition $g^{2}=1$ the second expression in (13) vanishes. These are the elements $g_{j}, j=1,2,3$. Since $\operatorname{tr}\left(i g_{j}\right)=1$ we have $T\left(i g_{j}\right)=0$. Nonzero ones are then

$$
T(i)=-\frac{1}{4|\vec{x}|^{4}}
$$

and

$$
T\left(g_{1}\right)=-\frac{1}{8\left(x_{2}^{2}+x_{3}^{2}\right)^{2}} .
$$

Remaining two terms $T\left(g_{2}\right), T\left(g_{3}\right)$ are obtained from the above equation by the cyclic replacements of coordinates.

Inserting the results from (16) to (21) into (12) we arrive at the energy density

$$
\begin{aligned}
T(x)=\frac{1}{12 \pi^{2}}\left[T\left(g_{4}\right)-T\left(i g_{4}\right)\right. & -T(i)] \\
& +\frac{1}{12 \pi^{2}}\left[\frac{1}{8} T\left(g_{1}\right)+2 T\left(g_{6}\right)-2 T\left(i g_{6}\right)+c . p .\right] .
\end{aligned}
$$


Here c.p. stands for cyclic permutations of coordinates.

\section{Wave Function and the Spectrum in the Conical Wedge}

In this section we briefly present the derivation of the wave function and the spectrum. We write the wave function in a similar fashion as the Green function as

$$
\Psi_{\vec{p}}(\vec{x})=\Omega \sum_{j=0}^{11}\left[e^{i\left(\vec{p}, g_{j} \vec{x}\right)}-e^{i\left(\vec{p}, i g_{j} \vec{x}\right)}\right]
$$

or

$$
\Psi_{\vec{p}}(\vec{x})=-8 i \Omega\left[\sin p_{1} x_{1} \sin p_{2} x_{2} \sin p_{3} x_{3}+\text { c.p. }\right]
$$

with $\Omega$ being the normalization. To obtain the spectrum we first observe that

$$
\Psi_{g_{j} \vec{p}}(\vec{x})=\Psi_{\vec{p}}(\vec{x}) \quad \Psi_{i \vec{p}}(\vec{x})=-\Psi_{\vec{p}}(\vec{x})
$$

which imply that the momentum $\vec{p}$ takes its values in the quotient space $R^{3} / G$ which is exactly the region $S$ of (15). In other words, the geometry in the momentum space is the replica of the geometry in the configuration space, the fact that can be used in deriving the spectra. Since

$$
\left.\Psi_{\vec{p}}(\vec{x})\right|_{\vec{p} \in P_{2}}=0
$$

we have to drop the values of $\vec{p}$ on the plane $P_{2}\left(p_{2}=0\right)$. The condition

$$
\left.\Psi_{\vec{p}}(\vec{x})\right|_{\vec{p} \in P_{1}}=\left.\Psi_{\vec{p}}(\vec{x})\right|_{g \vec{p} \in P_{3}}
$$

implies that momenta on the plane $P_{1}\left(p_{1}=p_{2}\right)$ are equivalent to the ones on the plane $P_{3}\left(p_{2}=p_{3}\right)$. It is sufficient to take into account momenta on one of these planes ( take for example values on $P_{1}$ ) and drop the ones on the other. Therefore the spectrum in the conical wedge is

$$
\Sigma_{0}=S \backslash(A \bigcup B)=\left\{p_{1} \geq p_{2}>0, \quad p_{3}>p_{2}>0\right\}
$$

where $A$ and $B$ are the boundaries of $S$ corresponding to $P_{2}$ and $P_{3}$ planes:

$$
\begin{gathered}
A=\left\{p_{1} \geq 0, \quad p_{2}=0 \quad p_{3} \geq 0\right\}, \\
B=\left\{p_{1} \geq p_{2} \geq 0, \quad p_{3}=p_{2} \geq 0\right\} .
\end{gathered}
$$

\section{Conical Cavity}

In addition to the planes of (11) consider two additional ones

$$
P_{4}: x_{1}=a, \quad P_{5}: x_{3}=a .
$$

The region we consider is then given by

$$
H=\left\{a \geq x_{1} \geq x_{2} \geq 0, \quad a \geq x_{3} \geq x_{2} \geq 0\right\} .
$$

Boundary conditions (26), (27) and new Dirichlet conditions on $P_{4}$ and $P_{5}$

$$
\left.\Psi_{\vec{p}}(\vec{x})\right|_{\vec{p} \in P_{4}}=\left.\Psi_{\vec{p}}(\vec{x})\right|_{\vec{p} \in P_{5}}=0
$$


imply that the geometry in hand is a conical cavity with the opening angle $\beta=\arcsin \frac{1}{3}$ and height $h=a \cos \beta=\frac{2 \sqrt{2}}{3} a$. The Dirichlet boundary conditions (33) quantize the momenta:

$$
p_{1}=\frac{\pi}{a} n, \quad p_{2}=\frac{\pi}{a} m, \quad p_{3}=\frac{\pi}{a} k
$$

where $n, m$ and $k$ are integers which subject to the condition (28) ( with $\vec{n}=(n, m, k))$

$$
\Sigma=\Sigma_{0} \bigcap Z^{3}=\left\{\vec{p}=\frac{\pi}{a} \vec{n}: \quad n \geq m>0, \quad k>m>0\right\} .
$$

The Green function can be written as

$$
G(x, y)=\sum_{m=1}^{\infty} \sum_{k=m+1}^{\infty} \sum_{n=m}^{\infty} \frac{e^{i \pi|\vec{p}|\left(x_{0}-y_{0}\right)}}{2|\vec{p}|} \Psi_{\vec{p}}(\vec{x}) \Psi_{\vec{p}}(\vec{y}) .
$$

The energy density we obtain is

$$
T(x)=\frac{\pi}{2 a} \sum_{m=1}^{\infty} \sum_{k=m+1}^{\infty} \sum_{n=m}^{\infty} \sqrt{n^{2}+m^{2}+k^{2}}\left|\Psi_{\vec{p}}(\vec{x})\right|^{2} .
$$

After integration $\int_{0}^{a} d x_{2} \int_{x_{2}}^{a} d x_{3} \int_{x_{2}}^{a} d x_{1}$ over the conical cavity $H$ we get the total energy

$$
E=\frac{\pi}{2 a} \sum_{\vec{n} \in \Sigma}|\vec{n}|=\frac{\pi}{48 a} \sum_{\vec{n} \in \Sigma} \sum_{g \in G}|g \vec{n}|=\frac{\pi}{48 a} \sum_{\vec{n} \in \bigcup_{g \Sigma}}|\vec{n}| .
$$

Since $\Sigma=S \backslash(A \cup B)) \cap Z^{3}$ and $S=R^{3} / G$ we have

$$
\bigcup_{g \in G} g \Sigma=Z^{3} \backslash C
$$

where

$$
C=\left(\bigcup_{g \in G} g(A \bigcup B)\right) \bigcap Z^{3}
$$

is the union of six planes in $Z^{3}: m=0, k=m$ and other four planes are obtained by cyclic permutations of $n, m$ and $k$ : .

$$
\sum_{\vec{n} \in C}|\vec{n}|=3 \sum_{n, m \in Z} \sqrt{n^{2}+m^{2}}+3 \sum_{n, m \in Z} \sqrt{2 n^{2}+m^{2}}
$$

(38) and (39) imply

$$
E=\frac{\pi}{48 a}\left(\sum_{\vec{n} \in Z^{3}}|\vec{n}|-\sum_{\vec{n} \in C}|\vec{n}|\right)
$$

or

$$
E=\frac{1}{3} E_{1}-\frac{1}{2} E_{2}-\frac{2+\sqrt{2}}{4} E_{3} \simeq \frac{0,085}{a} .
$$

Here $E_{1}, E_{2}$ and $E_{3}$ are the Casimir energies for the cube with sides $a$, for the rectangle with sides $a$ and $\frac{a}{\sqrt{2}}$; and, for the one dimensional system of 
length $a$ respectively [6]

$$
\begin{aligned}
& E_{1}=\frac{\pi}{2 a} \sum_{n, m, k=1}^{\infty} \sqrt{n^{2}+m^{2}+k^{2}} \simeq-\frac{0,015}{a}, \\
& E_{2}=\frac{\pi}{2 a} \sum_{n, m=1}^{\infty} \sqrt{n^{2}+2 m^{2}} \simeq \frac{0,045}{a} \\
& E_{3}=\frac{\pi}{2 a} \sum_{n=1}^{\infty} n \simeq-\frac{0,131}{a}
\end{aligned}
$$

\section{Discussion}

Examples of the Casimir energy calculations for 3-dimensional cavities are not plenty. The cubical [1, 6] and the spherical [7] cavities have been studied rather extensively. In this work we have studied a conical cavity with a particular opening angle. Similar restriction is true for the recently studied pyramidal geometry for which the angles at the vertices are not arbitrary [4. We do not have results in hand for the last two kind of geometries with arbitrary angles. Nevertheless it may be of interest to briefly review the known Casimir energy calculations ( for the massless scalar fields ) for the above mentioned geometries. To have better idea on the magnitudes we consider the cavities of comparable sizes:

For cube of sides $2 b$, we have negative value for the energy : $E_{c u b} \simeq-\frac{0,007}{b}$ [1, 6].

For spherical cavity of radius $b$ the Casimir energy is positive $E_{s p h} \simeq \frac{0,046}{b}$ [7].

Coming to the recently studied pyramidal cavity, let us first describe its position with respect to the above cube. One of the four vertices is located at the center of the cube, the remaining three at the center of a surface, and at the closest vertex and at the middle of the closest edge to this vertex [4]. The volume of this cavity is $\frac{b^{3}}{6}$ and the Casimir energy is again positive, $E_{p y r} \simeq \frac{0,069}{b}$.

For the present conical cavity of opening angle $2 \beta=2 \arcsin \frac{1}{3}$ and height $b$ ( i.e. $b=\frac{2 \sqrt{2}}{3} a$ ) which has the positive energy of (43) is $E_{\text {con }} \simeq \frac{0,08}{b}$.

To see the dependence of the magnitudes of the positive Casimir energies on the "shapes" of the cavities, let us compare the last three results for the "equal" volumes. If we consider the spherical, pyramidal and conical cavities with equal volumes we have the following ratios

$$
\begin{aligned}
E_{c o n} & \simeq 0,54 E_{s p h} \\
E_{p y r} & \simeq 0,51 E_{s p h} .
\end{aligned}
$$

It is not surprising that the conical and pyramidal geometries are very close to each other. 
Acknowledgments: We thank Turkish Academy of Sciences ( TUBA ) for its support, and D. Duru for the figures.

\section{Appendix A}

Tetrahedral group $T$ is the group of transformations which transforms cube into itself. The order of this group is 12 . We denote the identity element by $g_{0} . g_{1}, g_{2}$ and $g_{3}$ are rotations on $\pi$ around $\mathrm{x}, \mathrm{y}$ and z-axis:

$$
g_{1}=\left(\begin{array}{ccc}
1 & 0 & 0 \\
0 & -1 & 0 \\
0 & 0 & -1
\end{array}\right), g_{2}=\left(\begin{array}{ccc}
-1 & 0 & 0 \\
0 & 1 & 0 \\
0 & 0 & -1
\end{array}\right), g_{3}=\left(\begin{array}{ccc}
-1 & 0 & 0 \\
0 & -1 & 0 \\
0 & 0 & 1
\end{array}\right)
$$

$g_{4}$ and $g_{5}$ are rotations by $\frac{2 \pi}{3}$ and $\frac{4 \pi}{3}$ around the axis passing trough the origin and the point $(1,1,1)$ :

$$
g_{4}=\left(\begin{array}{lll}
0 & 0 & 1 \\
1 & 0 & 0 \\
0 & 1 & 0
\end{array}\right), g_{5}=\left(\begin{array}{lll}
0 & 1 & 0 \\
0 & 0 & 1 \\
1 & 0 & 0
\end{array}\right)
$$

$g_{6}$ and $g_{7}$ are rotations by $\frac{2 \pi}{3}$ and $\frac{4 \pi}{3}$ around the axis passing trough the origin and the point $(1,-1,1)$ :

$$
g_{6}=\left(\begin{array}{ccc}
0 & 0 & 1 \\
-1 & 0 & 0 \\
0 & -1 & 0
\end{array}\right), g_{7}=\left(\begin{array}{ccc}
0 & -1 & 0 \\
0 & 0 & -1 \\
1 & 0 & 0
\end{array}\right)
$$

$g_{8}$ and $g_{9}$ are rotations by $\frac{2 \pi}{3}$ and $\frac{4 \pi}{3}$ around the axis passing trough the origin and the point $(-1,1,1)$ :

$$
g_{8}=\left(\begin{array}{ccc}
0 & 0 & -1 \\
-1 & 0 & 0 \\
0 & 1 & 0
\end{array}\right), g_{9}=\left(\begin{array}{ccc}
0 & -1 & 0 \\
0 & 0 & 1 \\
-1 & 0 & 0
\end{array}\right)
$$

$g_{10}$ and $g_{11}$ are rotations by $\frac{2 \pi}{3}$ and $\frac{4 \pi}{3}$ around the axis passing trough the origin and the point $(1,1,-1)$ :

$$
g_{10}=\left(\begin{array}{ccc}
0 & 0 & -1 \\
1 & 0 & 0 \\
0 & -1 & 0
\end{array}\right), g_{11}=\left(\begin{array}{ccc}
0 & 1 & 0 \\
0 & 0 & -1 \\
-1 & 0 & 0
\end{array}\right)
$$

Let $G$ be a point group acting in the Euclidean space $R^{3}$. A closed subset $S$ of $R^{3}$ is called a fundamental domain of $G$ if $R^{3}$ is the union of conjugates of $S$, i.e.,

$$
R^{3}=\bigcup_{g \in G} g S
$$

and the intersection of any two conjugates has no interior. 
The fundamental domain of the group generated by the reflections $i g_{1}, i g_{2}$ and $i g_{3}$ with respect to $x_{1}=0, x_{2}=0$ and $x_{3}=0$ planes is the first quadrant in $R^{3}$. This is group of order 8 and divide $R^{3}$ into 8 equal parts. If one adds to this group the element $g_{4}$ we arrive at the group of order 24 which is the direct product of the tetrahedral group $T$ and inversion one $I$ generated by $i$. Rotation $g_{4}$ is three fold rotation. It divides the first quadrant into three equal parts. Therefore the fundamental domain for $T \times I$ is the region in the first quadrant between three planes (11).

For more details concerning finite groups we refer to $[8]$.

\section{References}

[1] V.M. Mostepanenko and N.N. Trunov "The Casimir Effect and its Applications", Oxford University Press, New York. (1997)

[2] D. Deutsch and P. Candelas, Phys. Rev., D20, 3063 (1979); J. S. Dowker and G. Kennedy, Jour. of Physics A11, 895 (1978).

[3] H. Ahmedov and I. H. Duru, J. Math. Phys. 45, 965 (2003)

[4] H. Ahmedov and I. H. Duru, "Casimir Energy for a Wedge with Three Surfaces and for a Pyramidal Region", arXiv:math-ph/0407030

[5] N.D. Birrel and P.C.V. Davies, "Quantum Fields in Curved Spaces", Cambridge University Press, (1982).

[6] S. G. Mamaev and N.N. Trunov, Theor. Math. Phys. 38, 228 (1979).

[7] T. H. Boyer, Phys. Rev. 174, 1764-76 (1968); B. Davies, Journal of Mathematical Physics, 13, 1324-8 (1972); R. Balian and B. Duplantier, Annals of Physics, 104, 300-35 (1978);J Schwinger, L.L. De Raad and K. A. Milton, Annals of Physics, 115, 1-23 (1978); 115, 388-403 (1978).

[8] See for example T. Janssen, "Crystallographic Groups", North Holland, Elsevier (1973). 
This figure "Figure1.jpg" is available in "jpg" format from: http://arxiv.org/ps/hep-th/0408199v1 
This figure "Figure2.jpg" is available in "jpg" format from: http://arxiv.org/ps/hep-th/0408199v1 